\title{
New results on charm decays and lifetimes from Fermilab experiment FOCUS
}

\author{
Gianluigi Boca on the behalf of the FOCUS collaboration* ${ }^{\dagger}$ \\ University of Pavia and INFN, via Bassi 6, I-27100 Pavia, Italy \\ E-mail: boca@pv.infn.it
}

\begin{abstract}
FOCUS is a very high statistics charm experiment, run at Fermilab in 19961997, which reconstructed over 1,000,000 charm events). In this paper some of the latest results in the decays of charm mesons and baryons will be reported : new decay channels, singly and doubly Cabibbo suppressed decays, lifetimes, 3-body Dalitz analysis.
\end{abstract}

\section{The FOCUS experiment}

The fixed target experiment FOCUS (E831) took data in 1996-1997[i]1ㄱ. A $\gamma$ beam impinged on a segmented $10 \%$ interaction length $\mathrm{BeO}$ target. A large aperture, forward spectrometer, with excellent vertex capabilities and particle identification, allowed the detection of the decay products of charmed mesons and baryons. The average $\gamma$ energy was $\simeq 180 \mathrm{GeV}$ for events that satisfied the trigger. Charged particles from the target were detected in four planes of microstrips interleaved with the segments of the target. More downstream, 12

\footnotetext{
${ }^{*}$ Speaker.

${ }^{\dagger}$ Coauthors: J.M.Link, M.Reyes, P.M.Yager (UC DAVIS); J.Anjos, I.Bediaga, C.Gobel, J.Magnin, A. Massafferri, J.M. de Miranda, I.M.Pepe, A.C. dos Reis, (CPBF, Rio de Janeiro); S.Carrillo, E.Casimiro, A.Sánchez-Hernández, C.Uribe, F.Vasquez (CINVESTAV, México City); L.Cinquini, J.P.Cumalat, B.O'Reilly, J.E.Ramirez, E.W.Vaandering (CU Boulder); J.N.Butler, H.W.K.Cheung, I.Gaines, P.H.Garbincius, L.A.Garren, E.Gottschalk, P.H.Kasper, A.E.Kreymer, R.Kuschke (Fermilab); S.Bianco, F.L.Fabbri, A.Zallo (INFN Frascati); C.Cawlfield, D.Y.Kim, A.Rahimi, J.Wiss (UI Champaign); R.Gardner, A.Kryemadhi (IU Bloomington); Y.S.Chung, J.S.Kang, B.R.Ko, J.W.Kwak, K.B.Lee, H.Park (Korea University, Seoul); G.Alimonti, M.Boschini, B.Caccianiga, P.D'Angelo, M.DiCorato, P.Dini, M.Giammarchi, P.Inzani, F.Leveraro, S.Malvezzi, D.Menasce, M.Mezzadri, L.Milazzo, L.Moroni, D.Pedrini, C.Pontoglio, F.Prelz, M.Rovere, S.Sala (INFN and Milano); T.F.Davenport III (UNC Asheville); L.Agostino, V.Arena, G.Boca, G.Bonomi, G.Gianini, G.Liguori, M.M.Merlo, D.Pantea, C.Riccardi, I.Segoni, P.Vitulo (INFN and Pavia); H.Hernandez, A.M.Lopez, H.Mendez, L.Mendez, E.Montiel, D.Olaya, A.Paris, J.Quinones, C.Rivera, W.Xiong, Y.Zhang (Mayaguez, Puerto Rico); J.R.Wilson (USC Columbia); K.Cho, T.Handler, R.Mitchell (UT Knoxville); D.Engh, M.Hosack, W.E.Johns, M.Nehring, P.D.Sheldon, K.Stenson, M.Webster (Vanderbilt); M.Sheaff (Wisconsin, Madison)
} 
planes of silicon microstrips were arranged in four stations with three views each. The whole microstrip detector provided an average resolution of $\sim 300 \mu \mathrm{m}$ in the direction along the beam and $\sim 6 \mu \mathrm{m}$ in the transverse directions. The momentum of the charged particles was detected by measuring the deflection in two analysing magnets and 20 planes of multiwire proportional chambers. Particle identification was provided by three threshold multicell Cerenkov counters for electrons, pions, kaons and protons while the muon identification was provided by scintillation counters and Resistive Plate Counters located behind large concrete and/or iron shieldings. Two electromagnetic calorimeters (a lead-glass calorimeter at small angles, a lead-scintillator sampling calorimeter at large angles) and a hadron calorimeter (a sampling iron-scintillator with tiles read out by WLS plastic fibers) where used to detect showers and provide signals to form the fast first level trigger.

\section{Results shown at this conference}

Presently FOCUS has the largest charm sample consisting of over a million reconstructed charm events. Here only some of the physics results will be mentioned : a) high statistics and/or new decay channels and relative branching ratios (BR) of charm mesons and baryons; b) lifetime measurements; c) Dalitz analysis of 3-body decays. Throughout this paper, whenever a decay is indicated, also the charge conjugate decay is implied.

\section{High statistics and/or new decay channels and relative branching ratios of charm mesons and baryons}

For almost all charm mesons new decay channels have been discovered or statistics has greatly improved compared to previous experiments.

1) $D^{+} \rightarrow K^{+} K^{+} K^{-}$and $D_{s}^{+} \rightarrow K^{+} K^{+} K^{-}$. These doubly Cabibbo suppressed (DCSD) and singly Cabibbo suppressed decays (SCSD) have never been observed before. They are particularly interesting since they cannot occur via simple spectator diagrams. Preliminary results of relative BR are $\frac{\Gamma\left(D^{+} \rightarrow K^{+} K^{+} K^{-}\right)}{\Gamma\left(D^{+} \rightarrow K^{-} \pi^{+} \pi^{+}\right)}=(0.14 \pm 0.02) \%$ and $\frac{\Gamma\left(D_{s}^{+} \rightarrow K^{+} K^{+} K^{-}\right)}{\Gamma\left(D_{s}^{+} \rightarrow K^{-} \pi^{+} \pi^{+}\right)}=(0.87 \pm 0.25) \%$ (no systematic errors yet).

2) $D^{+} \rightarrow K^{+} \pi^{+} \pi^{-}$and $D_{s}^{+} \rightarrow K^{+} \pi^{+} \pi^{-} ; \quad$ these decays are DCS and SCS respectively. The FOCUS signal consists of $433 \pm 48$ events for the $D^{+}$and $844 \pm 43$ events for the $D_{s}$, approximately 10 times the statistics of previous experiments E687 and E791. The BR relative to $K^{+} \pi^{+} \pi^{-}$is under study and soon the result will be available.

3) $D^{0} \rightarrow \pi^{+} \pi^{-} \pi^{+} \pi^{-} ; \quad$ a very clean signal of $7609 \pm 104$ evts and $S / N=12.6$ (largest statistics in the world) will allow the measurement of the relative $\operatorname{BR} \frac{\Gamma\left(D^{0} \rightarrow \pi^{+} \pi^{-} \pi^{+} \pi^{-}\right)}{\Gamma\left(D^{0} \rightarrow K^{-} \pi^{+} \pi^{+} \pi^{-}\right)}$ with a precision of $\sim 3 \%$.

4) $D^{ \pm} \rightarrow K_{s} \pi^{ \pm}$and $D^{ \pm} \rightarrow K_{s} K^{ \pm}$; the statistics in this channels allows to measure the relative $\mathrm{BR} \frac{\Gamma\left(D \rightarrow K_{s} \pi\right)}{\Gamma(D \rightarrow K \pi \pi \pi)}$ and $\frac{\Gamma\left(D \rightarrow K_{s} K\right)}{\Gamma(D \rightarrow K \pi \pi \pi)}$ with an unprecedented precision of $\sim 2 \%$, and the first measure of $A_{C P} \equiv\left[\frac{\Gamma\left(D^{+} \rightarrow K_{s} K^{+}\right)}{\Gamma\left(D^{+} \rightarrow K^{-} \pi^{+} \pi^{+} \pi^{-}\right)}-\frac{\Gamma\left(D^{-} \rightarrow K_{s} K^{-}\right)}{\Gamma\left(D^{-} \rightarrow K^{+} \pi^{+} \pi^{-} \pi^{-}\right)}\right] /\left[\frac{\Gamma\left(D^{+} \rightarrow K_{s} K^{+}\right)}{\Gamma\left(D^{+} \rightarrow K^{-} \pi^{+} \pi^{+} \pi^{-}\right)}+\right.$ $\left.\frac{\Gamma\left(D^{-} \rightarrow K_{s} K^{-}\right)}{\Gamma\left(D^{-} \rightarrow K^{+} \pi^{+} \pi^{-} \pi^{-}\right)}\right]$. These results have been submitted to PRL recently.

5) $D^{+} \rightarrow K_{s} K^{+} \pi^{+} \pi^{-}$and $D_{s}^{+} \rightarrow K_{s} K^{+} \pi^{+} \pi^{-} ; \quad$ these SCSD and Cabibbo favoured 
decays respectively, have never been observed before. The FOCUS relative BR result is $\frac{\Gamma\left(D^{+} \rightarrow K_{s} K^{+} \pi^{+} \pi^{-}\right)}{\Gamma\left(D^{+} \rightarrow K_{s} \pi^{+} \pi^{+} \pi^{-}\right)}=(5.62 \pm 0.39 \pm 0.40) \%$.

6) $D^{+} \rightarrow K_{s} K^{+} K^{-} \pi^{+}$; this decay has also never been observed before. A raw yield of $35 \pm 7$ events gives a relative BR of $\frac{\Gamma\left(D^{+} \rightarrow K_{s} K^{+} K^{-} \pi^{+}\right)}{\Gamma\left(D^{+} \rightarrow K_{s} \pi^{+} \pi^{+} \pi^{-}\right)}=(0.77 \pm 0.15 \pm 0.09) \%$.

7) $\Lambda_{c}^{+}$decay channels. $\quad$ FOCUS could collect the highest statistics in the world not only in the golden mode $p K \pi$ but also in the following decay channels : a) $\Lambda_{c} \rightarrow \Lambda^{0} \pi^{+} \pi^{+} \pi^{-}$; the raw yield of this signal is $1101 \pm 5$ events with $\frac{S}{N}=1.1$; b) $\Lambda_{c} \rightarrow p K_{s}$; the raw yield is $2822 \pm 127$ events with $\frac{S}{N}=0.5$; c) $\Lambda_{c} \rightarrow \Sigma^{+} \pi^{+} \pi^{-}$; the raw yield is $1418 \pm 63$; d) $\Lambda_{c} \rightarrow \Sigma^{-} \pi^{+} \pi^{+}$; the raw yield is $472 \pm 35$ events; e) $\Lambda_{c} \rightarrow \Sigma^{+} K^{+} K^{-}$; the raw yield is $111 \pm 16$ events with a large component of $\Lambda_{c} \rightarrow \Sigma^{+} \phi$ (about $56 \%$ of the raw signal). This decay are particularly interesting since they cannot be described by simple spectator diagrams

8) $\Xi_{c}^{+}$decay channels. The FOCUS large samples of $\Xi_{c}^{+}$consist, among the others, of : a) $\Xi_{c}^{+} \rightarrow p K^{-} \pi^{+}$; The raw yield is $202 \pm 35$ events. This is the only SCS decay channel of the $\Xi_{c}^{+}$known up to day and the only other observation is by experiment SELEX in 2000. The relative $\operatorname{BR} \frac{\Gamma\left(\Xi_{c}^{+} \rightarrow p K^{-} \pi^{+}\right)}{\Gamma\left(\Xi_{c}^{+} \rightarrow \Xi^{-} \pi^{+} \pi^{+}\right)}=0.234 \pm 0.047 \pm 0.022$ and it is in agreement with the Selex result. b) $\Xi_{c}^{+} \rightarrow \Omega^{-} K^{+} \pi^{+}$; this is a new decay according to PDG[[2] yield is $25 \pm 7$ events and $\frac{S}{N}=1.8$; c) $\Xi_{c}^{+} \rightarrow \Lambda^{0} K^{-} \pi^{+} \pi^{+}$; this channel has a raw yield of $137 \pm 25$ events; d) $\Xi_{c}^{+} \rightarrow \Sigma^{+} K^{-} \pi^{+}$; this signal consists of $181 \pm 18$ raw events.

9) $\Xi_{c}^{0}$ decay channels. $\quad$ FOCUS presently has two relatively high statistics channels : a) $\Xi_{c}^{0} \rightarrow \Xi^{-} \pi^{+}$; (94 \pm 14 events $)$ and b) $\Xi_{c}^{0} \rightarrow \Omega^{-} K^{+}$; this decay cannot be described at the tree level by a spectator diagram, but by an exchange diagram. The signal consists of $42 \pm 11$ events.

\section{Lifetime measurements}

The FOCUS collaboration is analysing the lifetime of all the singly charmed mesons and the $\Lambda_{c}, \Xi_{c}^{+}$and $\Xi_{c}^{0}$ baryons and, in the future, the lifetime of the $\Omega_{c}^{0}$. In this way it will be possible to determine better the lifetime hierarchy of the singly charmed particles, which is very important from the theoretical point of view.

1) $D^{0}$ and $D^{+}$lifetime;

preliminary results from this analysis show that the statistical errors are already better than the PDG[2] $]$ errors (which are 13 fs for the $D^{+}$and 2.8 fs for the $D^{0}$ ). The systematic errors are still being evaluated;

2) $D_{s}^{+}$lifetime;

the preliminary result, obtained with $50 \%$ of the total sample in the decay channel $D_{s}^{+} \rightarrow$ $\Phi \pi^{+}$is $\tau=506 \pm 8 \mathrm{fs}$, where the error is statistical only. This is in agreement with the present PDG average of $496_{-9}^{+10}$ fs; the statistical error is already comparable.

3) $\Lambda_{c}$ lifetime;

the preliminary result obtained using $80 \%$ of the whole data set is $\tau=204.5 \pm 3.4$ (stat.) fs and it is in agreement with and has better error than the present PDG[i $[\overline{2}]$ world average of $\tau=206 \pm 12$ (stat.)

4) $\Xi_{c}^{+}$lifetime; 
Table 1: Preliminary fit results for $D^{+} \rightarrow K^{+} K^{-} \pi^{+}$Dalitz analysis.

\begin{tabular}{|l|c|c|}
\hline & fit fraction & phase (Degrees) \\
\hline \hline$\overline{K^{0 *}}(890)$ & $0.30 \pm 0.01$ & 0 (fixed) \\
$\overline{K^{0 *}}(1430)$ & $0.38 \pm 0.01$ & $65 \pm 3$ \\
$\phi(1020)$ & $0.28 \pm 0.01$ & $-179 \pm 4$ \\
\hline
\end{tabular}

Table 2: Preliminary fit results for the $D_{s}^{+} \rightarrow K^{+} K^{-} \pi^{+}$Dalitz analysis.

\begin{tabular}{|l|c|c|}
\hline & fit fraction & phase (Degrees) \\
\hline \hline$\overline{K^{0 *}}(890)$ & $0.44 \pm 0.01$ & 0 (fixed) \\
$\overline{K^{0 *}}(1430)$ & $0.06 \pm 0.01$ & $114 \pm 5$ \\
$\phi(1020)$ & $0.45 \pm 0.01$ & $148 \pm 4$ \\
$f_{0}(980)$ & $0.16 \pm 0.01$ & $135 \pm 4$ \\
$f_{1}(1710)$ & $0.04 \pm 0.01$ & $106 \pm 8$ \\
\hline
\end{tabular}

the decay channels used in this analysis are $\Xi_{c}^{+} \rightarrow \Xi^{-} \pi^{+} \pi^{+}, \Xi_{c}^{+} \rightarrow \Sigma^{+} K^{-} \pi^{+}, \Xi_{c}^{+} \rightarrow$ $\Lambda K^{-} \pi^{+} \pi^{+}$and $\Xi_{c}^{+} \rightarrow p K^{-} \pi^{-}$. We measure $\tau=0.439 \pm 0.022$ (stat.) \pm 0.009 (sys.) ps. The PDG[플 world average is presently $330_{-40}^{+60}$ fs which is compatible within $2 \sigma$ with FOCUS result;

5) $\Xi_{c}^{0}$ lifetime;

a preliminary result obtained using only the decay channel $\Xi_{c}^{0} \rightarrow \Xi^{-} \pi^{+}$is $\tau=124 \pm$ 10 (stat.) fs, in agreement with the PDG[2] average of $\tau=98_{-15}^{+23}$ fs.

\section{Dalitz analysis}

FOCUS has presently the largest statistics in the world for all 3-body decay channels of the $D$ mesons. Here preliminary results from $D^{+} \rightarrow K^{+} K^{-} \pi^{+}, D_{s}^{+} \rightarrow K^{+} K^{-} \pi^{+}$, $D^{0} \rightarrow K_{s} K^{+} K^{-}, D^{+} \rightarrow \pi^{+} \pi^{+} \pi^{-}$and $D_{s}^{+} \rightarrow \pi^{+} \pi^{+} \pi^{-}$are presented.

1) Dalitz analysis of $D^{+} \rightarrow K^{+} K^{-} \pi^{+}$. The Dalitz plot consists of $\sim 6600$ events. Preliminary results are summarized in table $\overline{1}_{1}^{1}$. Three resonances dominate, the $\overline{K^{0 *}}(890)$, the $\phi$ and the $\overline{K^{0 *}}(1430)$. The absence of the non-resonant component in the fit fraction indicates that this is essentially a two-body decay. This preliminary results are in agreement with the previous experimental results by E687.

2) Dalitz analysis of $D_{s}^{+} \rightarrow K^{+} K^{-} \pi^{+}$. The Dalitz plot consists of $\sim 7500$ events. The preliminary fit results are summarized in table ${ }_{2}^{-}$Also this decay is strongly dominated by the $\overline{K^{0 *}}(890)$ and $\phi$. The fit fraction of $\overline{K^{0 *}}(1430)$ in this case is small and a sizeable fit fraction od $f_{0}(980)$ is present. This result is in agreement with the most recent experimental published result (E687).

3) Dalitz analysis of $D^{0} \rightarrow K_{s} K^{+} K^{-}$. In this channel the $D^{0}$ is tagged with $D^{*}$ to determine its charm content. This decay channel cannot be described by a simple spectator diagram and diagrams in which intermediate states couple both to $K^{+} K^{-}$and $\pi^{+} \pi^{-}$(like $f_{0}(980)$ or $\left.a_{0}^{0}(980)\right)$ could play an important role. In fact in table $\underline{3}_{-1}^{1}$ (preliminary results), 
Table 3: Preliminary fit results for $D^{0} \rightarrow K_{s} K^{+} K^{-}$Dalitz analysis.

\begin{tabular}{|l|c|c|}
\hline & fit fraction & phase (Degrees) \\
\hline \hline$f_{0}(980)$ or $a_{0}^{0}(980)$ & $0.692 \pm 0.044 \pm 0.02$ & 0 (fixed) \\
$\phi(1020)$ & $0.462 \pm 0.036 \pm 0.02$ & $-87 \pm 11 \pm 7$ \\
$a_{0}^{+}(980)$ & $0.124 \pm 0.034 \pm 0.02$ & $140 \pm 19 \pm 6$ \\
\hline
\end{tabular}

Table 4: Preliminary fit results for the $D_{s} \rightarrow \pi^{+} \pi^{+} \pi^{-}$Dalitz analysis.

\begin{tabular}{|l|c|c|}
\hline & fit fraction & phase (Degrees) \\
\hline \hline NR & $0.2345 \pm 0.0484$ & $244 \pm 6$ \\
$\rho^{0}(770)$ & $0.0058 \pm 0.0076$ & $229 \pm 60$ \\
$f_{0}(980)$ & $0.9057 \pm 0.0432$ & 0 (fixed) \\
$f_{2}(1270)$ & $0.1020 \pm 0.0143$ & $141 \pm 8$ \\
$S_{0}(1475)$ & $0.1922 \pm 0.0351$ & $70 \pm 6$ \\
$\rho^{0}(1450)$ & $0.0354 \pm 0.0088$ & $200 \pm 23$ \\
\hline
\end{tabular}

Table 5: Preliminary fit results for the $D^{+} \rightarrow \pi^{+} \pi^{+} \pi^{-}$Dalitz analysis.

\begin{tabular}{|l|c|c|}
\hline & fit fraction & phase (Degrees) \\
\hline \hline NR & $0.098 \pm 0.043$ & 0 (fixed) \\
$\rho^{0}(770)$ & $0.328 \pm 0.038$ & $63 \pm 17$ \\
$f_{0}(400)$ & $0.189 \pm 0.052$ & $-97 \pm 31$ \\
$f_{0}(980)$ & $0.067 \pm 0.015$ & $-146 \pm 18$ \\
$f_{2}(1270)$ & $0.123 \pm 0.021$ & $-213 \pm 18$ \\
$S_{0}(1475)$ & $0.018 \pm 0.012$ & $242 \pm 26$ \\
\hline
\end{tabular}

beside the strong presence of $\phi$ one can actually see a large fit fraction for a $f_{0}(980)$ and/or $a_{0}^{0}(980)$ resonance and the presence of $a_{0}^{+}(980)$.

4) Dalitz analysis of $D_{s}^{+} \rightarrow \pi^{+} \pi^{+} \pi^{-}$. The Dalitz plot consists of 1475 events. In this channel one expects the presence of resonances coupling to both $K \bar{K}$ and $\pi \pi$ to account for the absence of strange quarks in the final state $(3 \pi)$. As one can see from the preliminary results shown in table $\underline{4 i}_{1}^{\overline{4}}$ one can actually note a large fit fraction for a $f_{0}(980)$ and conversely a very low fit fraction of $\rho^{0}(770)$.

5) Dalitz analysis of $D^{+} \rightarrow \pi^{+} \pi^{+} \pi^{-}$. The Dalitz plot consists of 1527 events and presently it is the largest sample in the world. It is a CSD and the preliminary results are summarized in table with respect to the previous Dalitz plot and there is a large fit fraction for the $\rho^{0}(770)$.

\section{References}

[1] The FOCUS spectrometer is an upgraded version of the Fermilab E687 spectrometer which is described in P.L.Frabetti et al., Nucl. Instrum. Meth. A320, 519 (1992).

[2] Review of Particle Physics, The European Physical Journal C 15, 1 (2000). 